\title{
ON ISOMETRIC IMMERSIONS IN EUCLIDEAN SPACE OF MANIFOLDS WITH NON-NEGATIVE SECTIONAL CURVATURES $\left({ }^{1}\right)$
}

\author{
BY \\ PHILIP HARTMAN
}

1. This paper deals with certain isometric immersions $S$ of a complete $d$-dimensional manifold $M=M^{d}$ in a Euclidean space $E^{d+\delta}, \delta>0$ :

$$
\psi: M^{d} \rightarrow E^{d+\delta}, \quad S=\psi\left(M^{d}\right) .
$$

One of the main results will be the following:

THEOREM (*). Let $M=M^{d}$ be a complete Riemann manifold of class $C^{2}$ such that all 2-dimensional sections have non-negative curvatures. Let (1.1) be a $C^{2}$ isometric immersion of $M^{d}$ in $E^{d+\delta}, \delta>0$, such that the relative nullity function $\nu$ is a positive constant. Then $S$ is $\nu$-cylindrical.

The relative nullity $\nu(m), m \in M^{d}$ and $0 \leqq \nu(m) \leqq d$, is defined by Chern and Kuiper [2] (and for $\delta=1$ reduces to the nullity of the second fundamental matrix); cf. $\$ 3$ below.

The immersion (1.1) is said to be $\nu$-cylindrical if $M^{d}, \psi$, and $E^{d+\delta}$ can be expressed as products: $M^{d}=M^{d-\nu} \times E^{\nu}, \psi=\bar{\psi} \times 1$, and $E^{d+\delta}=E^{d-\nu+\delta}$ $\times E^{\nu}$, where $M^{d-\nu}$ is a complete Riemann manifold, $\bar{\psi}: M^{d-\nu} \rightarrow E^{d-\nu+\delta}$ is an isometric immersion and 1 is the identity map on $E^{\nu} . M^{d-\nu}$ and its immersion $\bar{\psi}$ are allowed to be of class $C^{1}$.

Theorem $\left({ }^{*}\right)$ is a consequence of Lemmas 3.1 and 4.1, below.

$\left({ }^{*}\right)$ generalizes a result of $O^{\prime} N e i l l[5]$ who supposes that $M$ is flat and makes the superfluous assumption that the "relative curvature of $\psi$ is 0 ." For the case of hypersurfaces $(\delta=1),\left(^{*}\right)$ is a particular case of a theorem of Sacksteder [6] which does not contain the condition that $\nu(m)$ is constant and which has a stronger conclusion. For the case of a flat $M$ and $\delta=1,\left(^{*}\right)$ is also contained in a result of Hartman and Nirenberg [4]. In the latter, the assumptions " $M$ flat" and " $\delta=1$ " imply that $d-1$ $\leqq \nu(m) \leqq d$. It will be clear from the proof that an analogue of $\left(^{*}\right)$ is correct if the assumption that " $\nu(m)$ is a positive constant" is replaced by the assumption that " $d-1 \leqq \nu(m) \leqq d$," in which case $M$ is necessarily flat. Professor Nirenberg has pointed out to me that this analogue can be deduced directly from our result in [4].

The problem of removing the assumption in $\left(^{*}\right)$ that $\nu(m)$ is a constant will remain open (when $\delta>1$ ).

Received by the editors December 3, 1963.

(1) This research was supported by the Air Force Office of Scientific Research. 
The proof of $\left({ }^{*}\right)$ depends in part on a generalization of the implicit function theorem, in the large, for gradient mappings in Lemma 2 of Chern and Lashoff [3] (or, equivalently, Lemma 2 of Hartman and Nirenberg [4]). This generalization involves simultaneous gradient mappings and is given in $\$ 2$.

An Appendix deals with a further generalization of this implicit function theorem.

2. Let $D$ be an open set in a Euclidean $d$-dimensional space of points $u=\left(u^{1}, \cdots, u^{d}\right)$. By a $\nu$-dimensional plane section $\pi$, of $D$ through a point $u \in D$ is meant the connected component, containing $u$, of the intersection of $D$ and a $\nu$-dimensional plane through $u$.

Let $P_{p}(u)=\left(P_{p}^{1}(u), \cdots, P_{p}^{d}(u)\right)$, where $p=1, \cdots, \delta$, be a vector function of class $C^{1}$ on a domain $D$ such that $u \rightarrow P_{p}(u)$ is a gradient mapping, i.e.,

$$
\omega_{p}=P_{p}(u) \cdot d u=P_{p}^{i}(u) \cdot d u^{i}
$$

is closed, so that

$$
d \omega_{p}=0, \quad p=1, \cdots, \delta .
$$

Let $J_{p}(u)=\left(\partial P_{p}^{i} / \partial u^{j}\right)$, where $i, j=1, \ldots, d$, be the $d \times d$ Jacobian matrix of the map $u \rightarrow P_{p}(u)$. Let $P(u)=\left(P_{1}, \cdots, P_{\delta}\right)=\left(P_{1}^{1}, \ldots, P_{1}^{d}, P_{2}^{1}, \ldots, P_{\delta}^{d}\right)$ be the $d \delta$-dimensional vector function and $J(u)$ the Jacobian matrix $(\partial P / \partial u)$ with $d$ columns and $\delta d$ rows:

$$
J=\left(\begin{array}{c}
J_{1} \\
\vdots \\
J_{\delta}
\end{array}\right) .
$$

Let $\rho(u)=\operatorname{rank} J(u)$ and $\rho^{*}\left(u_{0}\right)=\limsup \rho(u)$ as $u \rightarrow u_{0}$; correspondingly, $\nu(u)=$ nullity $J(u)=d-\rho(u)$ and $\nu^{*}\left(u_{0}\right)=d-\rho^{*}\left(u_{0}\right)=\liminf \nu(u)$ as $u \rightarrow u_{0}$. Finally, let $D$, be the open subset of $D$ defined by

$$
D_{\nu}=\left\{u: \nu^{*}(u) \geqq \nu\right\} .
$$

Lemma 2.1. Let $P_{1}(u), \ldots, P_{\delta}(u)$ be d-dimensional vector functions of class $C^{1}$ on $D$ such that (2.1) satisfies (2.2). Let $u_{0} \in D$ and $\nu\left(u_{0}\right), \nu^{*}\left(u_{0}\right)$ have a common value $\nu$. Then $P(u)=\left(P_{1}(u), \ldots, P_{\delta}(u)\right)$ is constant on a $\nu$-dimensional plane section $\pi_{v}\left(u_{0}\right)$ of $D_{v}$ through $u_{0}$. Furthermore, for all points $u$ near $u_{0}, P(u)=P\left(u_{0}\right)$ if and only if $u \in \pi_{\nu}\left(u_{0}\right)$. Finally, $\nu(u)=\nu^{*}(u)$ $=\nu$ for all $u \in \pi_{\nu}\left(u_{0}\right)$.

As in [4], this has the following consequence:

Corollary 2.1. If $\nu^{*}\left(u_{0}\right)=\nu$ at a point $u_{0} \in D$, then $P(u)$ is constant on a $\nu$-dimensional plane section $\pi_{\nu}\left(u_{0}\right)$ of $D_{\nu}$ through $u_{0}$. Also, $u \in \pi_{\nu}$ implies that $\nu^{*}(u)=\nu$ and that either $\nu(u)=\nu$ or $\nu(u)>\nu$ according as $\nu\left(u_{0}\right)=\nu$ or $\nu\left(u_{0}\right)>\nu$. 
While $\pi_{\nu}\left(u_{0}\right)$ is unique in Lemma 2.1, it need not be unique in Corollary 2.1. It is unique in Corollary 2.1 if $\nu^{*}(u)=d-1$.

Below, repeated indices indicate summation with the following ranges for the different indices:

$$
\begin{array}{ll}
1 \leqq h, i, j, k \leqq d ; & 1 \leqq \alpha, \beta, \gamma \leqq \rho ; \\
\rho+1 \leqq \kappa, \lambda \leqq d ; & 1 \leqq p, q, r \leqq \delta .
\end{array}
$$

Proof of Lemma 2.1. Let $J_{p \rho}(u)$ denote the $\rho \times \rho$ Jacobian matrix $\left(\partial P_{p}^{\alpha} / \partial u^{\beta}\right)$, where $\alpha, \beta=1, \cdots, \rho=d-\nu$, in the upper left corner of $J_{p}$ and let $J^{p}(u)$ denote the matrix

$$
J^{\rho}=\left(\begin{array}{c}
J_{1 \rho} \\
\vdots \\
J_{\delta \rho}^{-}
\end{array}\right)
$$

with $\rho$ columns and. $\delta \rho$ rows.

Let the $j_{1}$ st, $j_{2}$ nd, $\cdots, j_{\text {, th }}$ columns of $J\left(u_{0}\right)$ be linearly independent. Let $U$ be a $d \times d$ permutation (orthogonal) matrix such that the effect of multiplying any $d \times d$ matrix $A$ on the right by $U$ to give $A U$ is to move the $j_{1}$ st, $\cdots, j_{\nu}$, th columns of $A$ into the 1 st, $\cdots, \nu$ th places. Multiplication of $A$ on the left by $U^{*}$ to give $U^{*} A$ moves the $j_{1}$ st, $\cdots, j_{v}$ th rows of $A$ into the 1 st, $\cdots, \nu$ th positions, respectively.

Let $U u$ be renamed $u$ and $U^{*} P_{p}(U u)$ be called $P_{p}(u)$. Then $u \rightarrow P_{p}(u)$ satisfies (2.1), (2.2) and the first $\nu$ columns of $J(u)$ are linearly independent at $u=u_{0}$, hence, for $u$ near $u_{0}$. Since $\rho\left(u_{0}\right)=\rho^{*}\left(u_{0}\right)=\rho$, the last $\nu=d-\rho$ columns of $J(u)$ are linear combinations of the first $\rho$ columns for $u$ near $u_{0}$. In particular, the last $\nu=d-\rho$ columns of $J_{p}(u)$ are linear combinations of the first $\rho$ columns of $J_{p}(u)$ for $p=1, \cdots, \delta$. The condition (2.2) implies that the Jacobian matrix $J_{p}(u)$ is symmetric and so the last $\nu$ rows of $J_{p}(u)$ are linear combinations of the first $\rho$ rows. It follows that the rank of the matrix $J^{o}(u)$ in (2.3) is $\rho$.

Thus, $J^{\rho}(u)$ has $\rho$ linearly independent rows, i.e., there exist $\rho$ pairs of indices $(p(\alpha), i(\alpha))$, where $1 \leqq p(\alpha) \leqq \delta, 1 \leqq i(\alpha) \leqq \rho$ and $\alpha=1, \cdots, \rho$, such that $\operatorname{det}\left(\partial P_{p(\alpha)}^{i(\alpha)}(u) / \partial u^{\beta}\right)_{\alpha, \beta=1, \cdots, \beta} \neq 0$ at $u=u_{0}$. Introduce the mapping $u \rightarrow v$, defined by

(2.4) $v^{\alpha}=P_{p(\alpha)}^{i(a)}(u) \quad$ for $\alpha=1, \cdots, \rho$ and $v^{\kappa}=u^{\kappa} \quad$ for $\kappa=\rho+1, \cdots, d$, so that the Jacobian $\operatorname{det}(\partial v / \partial u) \neq 0$ at $u=u_{0}$. Let $v_{0}$ correspond to $u_{0}$ and let $u=u(v)$ be the local inverse of the map (2.4).

The above description of the linear dependence of the rows of $J_{p}(u)$ and the assumption $\rho(u)=\rho^{*}(u)=\rho$ for $u$ near $u_{0}$ imply that $P_{p}^{i}(u(v))$, for $p=1, \cdots, \delta$ and $i=1, \cdots, d$, is a function, say $\bar{P}_{p}^{i}(v)$, of $\left(v^{1}, \cdots, v^{\rho}\right)$. For

$$
\partial \bar{P}_{p}^{i} / \partial v^{k}=\left(\partial P_{p}^{i} / \partial u^{j}\right)\left(\partial u^{j} / \partial v^{k}\right)
$$


and if $\lambda>\rho$ and $u$ (or $v$ ) is fixed, there are numbers $c_{\lambda \beta}, \beta=1, \cdots, \rho$, such that

$$
\partial P_{p}^{i} / \partial u^{\lambda}=c_{\lambda \beta} \partial P_{p}^{i} / \partial u^{\beta} \quad \text { för } i=1, \cdots, d .
$$

Hence $\partial u^{\lambda} / \partial v^{\alpha}=\delta_{\kappa}^{\lambda}$ implies that

$$
\partial \bar{P}_{p}^{i} / \partial v^{\kappa}=\left(\partial P_{p}^{i} / \partial u^{\beta}\right)\left(\partial u^{\beta} / \partial v^{\kappa}+c_{k \beta}\right) .
$$

The choice $(p, i)=(p(\alpha), i(\alpha))$ for $\alpha=1, \cdots, \rho$, makes the left side zero and hence, $\partial u^{\beta} / \partial v^{\kappa}+c_{\kappa \beta}=0$ for $\beta=1, \cdots, \rho$ and $\kappa=\rho+1, \cdots, d$. Consequently $\partial \bar{P}_{p}^{i} / \partial v^{\kappa}=0$ for $p=1, \cdots, \delta ; i=1, \cdots, d$; and $\kappa=\rho+1, \cdots, d$.

Since the Pfaffian (2.1) is closed, it follows that the Pfaffian

$$
\omega_{p 0}=u^{i} d P_{p}^{i}(u)=d\left(u^{i} P_{p}^{i}\right)-\omega_{p}
$$

is also closed. Introducing $v$ as independent variable leaves $\omega_{p 0}$ closed and shows that $\omega_{p 0}$ is of the form

$$
\omega_{p 0}=\left(u^{i} \partial \bar{P}_{p}^{i} / \partial v^{\alpha}\right) d v^{\alpha} .
$$

Corresponding to $v$ near $v_{0}$, there is a scalar function $f_{p}\left(v^{1}, \cdots, v^{\rho}\right)$ of class $C^{1}$ such that $\omega_{p 0}=d f_{p}$. Hence

$$
u^{i} \partial \bar{P}_{p}^{i}(v) / \partial v^{\alpha}=b_{p \alpha}\left(v^{1}, \cdots, v^{\rho}\right) \quad \text { for } p=1, \cdots, \delta \text { and } \alpha=1, \cdots, \rho
$$

where $b_{p \alpha}=\partial f_{p} / \partial v^{\alpha}$ is continuous.

It will be verified that the $\delta \rho$ equations (2.5) contain a set of $\rho$ equations such that the matrix of coefficients of $u^{1}, \cdots, u^{\rho}$ is nonsingular and that the remaining equations of (2.5) are consequences of these. If this is granted for a moment, these $\rho$ equations can be solved for $u^{1}, \cdots, u^{\rho}$ to give a result of the form

$$
\begin{aligned}
& u^{\alpha}=a^{\alpha \kappa}\left(v^{1}, \cdots, v^{\rho}\right) v^{\kappa}+b^{\alpha}\left(v^{1}, \cdots, v^{\rho}\right) \quad \text { for } \alpha=1, \cdots, \rho, \\
& u^{\kappa}=v^{\kappa} \quad \text { for } \kappa=\rho+1, \cdots, d .
\end{aligned}
$$

Since (2.6) is the inverse of (2.4), it follows that the functions $a^{\alpha \kappa}, b^{\alpha}$ are of class $C^{1}$.

In order to see that (2.5) can be solved to give (2.6), write $\partial \bar{P}_{p}^{i} / \partial v^{\alpha}$ as the sum $\left(\partial P_{p}^{i} / \partial u^{\beta}\right)\left(\partial u^{\beta} / \partial v^{\alpha}\right)$. Thus, if (2.5) is multiplied by $\partial v^{\alpha} / \partial u^{\gamma}$ and the result summed for $\alpha=1, \cdots, \rho$, it follows that (2.5) is equivalent to

$$
u^{\alpha} \partial P_{p}^{\gamma} / \partial u^{\alpha}+u^{\kappa} \partial P_{p}^{\alpha} / \partial u^{\gamma}=b_{p \alpha} \partial v^{\alpha} / \partial u^{\gamma}
$$

for $p=1, \cdots, \delta$ and $\gamma=1, \cdots, \rho$, where $\alpha$ is a summation index over 1 , $\cdots, \rho$ and $\kappa$ over $1, \cdots, \nu$ and $\partial P_{p}^{\gamma} / \partial u^{\alpha}=\partial P_{p}^{\alpha} / \partial u^{\gamma}$.

The normalization of $J(u)$ shows that the $\delta \rho$ equations (2.7) are equivalent to the $\rho$ equations corresponding to $(p, \gamma)=(p(\beta), i(\beta))$ for $\beta=1$, $\cdots, \rho$. The matrix of coefficients of $u^{1}, \cdots, u^{\rho}$ in these $\rho$ equations is $\left(\partial P_{p(\beta)}^{i(\beta)} / \partial u^{\alpha}\right)_{\alpha, \beta=1, \cdots, \rho}$, which is nonsingular. 
Since $P_{p}^{i}(u)=\vec{P}_{p}^{i}(v)$ is a function of $\left(v^{1}, \cdots, v^{\circ}\right)$, the relations (2.6) imply the "local" part of Lemma 2.1; namely, that there exists a $\nu$-plane $\pi_{\nu}$ through $u_{0}$ such that $u$ near $u_{0}$ is on $\pi_{v}$ if and only if $P(u)=P\left(u_{0}\right)$.

The proof of the remainder of Lemma 2.1 is similar to that of Lemma 2 in [4] and will only be indicated. Substitution of (2.6) into the first part of (2.4) and differentiation with respect to $v^{\beta}$ gives

$$
\delta_{\alpha \beta}=\left(\partial P_{p(\alpha)}^{i(\alpha)} / \partial u^{\gamma}\right)\left(v^{\alpha} \partial a^{\gamma \kappa} / \partial v^{\beta}+\partial b^{\gamma} / \partial v^{\gamma}\right)
$$

for $\alpha, \beta=1, \cdots, \rho$; hence

$$
1=\operatorname{det}\left(\partial P_{p(\alpha)}^{i(\alpha)} / \partial u^{\gamma}\right) \operatorname{det}\left(v^{\alpha} \partial a^{\gamma \kappa} / \partial v^{\beta}+\partial b^{\gamma} / \partial v^{\beta}\right) .
$$

This shows that as $u \in D_{\text {v }}$ moves continuously from $u_{0}$ on the $\nu$-plane (2.6), where $v^{\alpha}$ is constant, one cannot reach a first point $u$ where

$$
\operatorname{det}\left(\partial P_{p(\alpha)}^{i(\alpha)} / \partial u^{\beta}\right)=0 .
$$

Thus the arguments above give Lemma 2.1.

REMARK. If $P_{p}(u)$ is of class $C^{t}, t \geqq 1$, then the change of coordinates $u \rightarrow v$ in the above proof is of class $C^{t}$.

3. Consider a piece of $d$-dimensional surface $S: X=X(u)$ of class $C^{2}$ in a $(d+\delta)$-dimensional Euclidean space $E^{d+\delta}$ where $u=\left(u^{1}, \cdots, u^{d}\right)$, $X=\left(X^{1}, \ldots, X^{d+\delta}\right)$, and $X(u)$ is of class $C^{2}$ on an open connected $u$-set $D$ such that rank $\left(\partial X^{\tau} / \partial u^{i}\right)$ is $d$.

Let $X_{i}=\partial X / \partial u^{i}, X_{i j}=\partial^{2} X / \partial u^{i} \partial u^{j}$, etc. If $N$ is a normal vector to $S$ at a point $u$ (i.e., $X(u)$ ), there is an associated $d \times d$ second fundamental matrix $\left(h_{i j}(u ; N)\right)$, where $h_{i j}(u ; N)$ is the Euclidean scalar product $X_{i j}(u) \cdot N$. Let $\pi(u ; N)$ denote the null space of $\left(h_{i j}(u ; N)\right)$, i.e., the set of vectors $y=\left(y^{i}, \cdots, y^{d}\right)$ satisfying $h_{i j} y^{j}=0$ for $i=1, \cdots, d$. Let $\pi(u)=\bigcap_{\pi(u ; N)}$ where the intersection is taken over all normals or, equivalently, $\pi(u)$

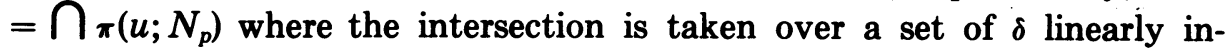
dependent normal vectors $N_{1}, \cdots, N_{\delta}$. The integer $\nu(u)=\operatorname{dim} \pi(u)$ is called the relative nullity of $S$ at $u[2]$. A vector $y \neq 0$ in $\pi(u)$ will be said to be in a trivial asymptotic direction at $u$.

Let $\nu^{*}\left(u_{0}\right)=\liminf \nu(u), u \rightarrow u_{0}$. The subset $D_{\tau}=\left\{u: \nu^{*}(u) \geqq \tau\right\}$ of $D$ is open.

Lemma 3.1. Let $M=M^{d}$ be a d-dimensional Riemann manifold of class $C^{2}$ and $S=\psi(M)$, where $\psi: M \rightarrow E^{d+\delta}$ is a $C^{2}$ isometric immersion of $M$ in the Euclidean space $E^{d+\delta}$. For a point $m \in M$, let $\nu(m)$ be the relative nullity of $S$ at $m$ (i.e., at $\psi(m)) ; \nu^{*}\left(m_{0}\right)=\liminf \nu(m), \quad m \rightarrow m_{0} ;$ and $M(\tau)$ the submanifold of $M$ consisting of points $\left\{m: \nu^{*}(m) \geqq \tau\right\}$.

(i) Then, for any point $m_{0} \in M$, there is a (not necessarily unique) maximal, totally geodesic submanifold $M^{\nu^{*}\left(m_{0}\right)}$ of $\left.M_{\left(\nu^{*}\right.}\left(m_{0}\right)\right)$ containing $m_{0}$, of dimension $\nu^{*}\left(m_{0}\right)$, which $\psi$ maps isometrically onto a subset of a $\nu^{*}\left(m_{0}\right)$-plane 
$\pi$ in $E^{d+\delta}$.

(ii) The space of normal vectors to $S$ at a point $\psi(m) \in \pi, m \in M^{*^{*}\left(m_{0}\right)}$, is independent of $m$.

(iii) If $m \in M^{\nu^{*}\left(m_{0}\right)}$, then $\nu^{*}(m) \geqq \nu(m)$ according as $\nu^{*}\left(m_{0}\right) \geqq \nu\left(m_{0}\right)$.

(iv) If $\nu\left(m_{0}\right)=\nu^{*}\left(m_{0}\right)$, then $M^{\nu^{*}\left(m_{0}\right)}$ is unique and $m$ near $m_{0}$ is on $M^{\nu^{*}\left(m_{0}\right)}$ if and only if the space of normal vectors at $\psi(m)$ is the same as that at $\psi\left(m_{0}\right)$.

(v) Finally, if $M$ is complete and $\nu\left(m_{0}\right)=\min \nu(m)$ for $m \in M$ (so that $\left.M\left(\nu\left(m_{0}\right)\right)=M\right)$, then $M^{\nu\left(m_{0}\right)}$ is complete and $\pi=\psi\left(M^{\nu\left(m_{0}\right)}\right)$ is a $\nu\left(m_{0}\right)$-dimensional plane in $E^{d+\delta}$.

The case $\delta=1$ is contained in [4]. For arbitrary $\delta>0$, under the additional assumption that $M$ is flat, the last part of Lemma 3.1 is contained in [5]. For a flat $M, d-\delta \leqq \nu(m) \leqq d$ holds [2]; so that, in this case, $\delta<d$ implies that $\nu(m)>0$ and Lemma 3.1 is not trivially true.

Proof. On introducing suitable local coordinates $u=\left(u^{1}, \cdots, u^{d}\right)$ on $M$ at $m_{0}$ and a suitable choice of rectangular coordinates $X=(u, x)=\left(u^{1}\right.$, $\left.\cdots, u^{d}, x^{1}, \cdots, x^{\delta}\right)$ in $E^{d+\delta}$, the immersion $\psi$, for $m$ near $m_{0}$, can be represented in the form

$$
x^{p}=\phi_{p}(u) \quad \text { for } p=1, \cdots, \delta,
$$

where $\phi_{p}$ is a real-valued $C^{2}$ function of $u=\left(u^{1}, \cdots, u^{d}\right)$. Let $P_{p}(u)$ $=\left(\partial \phi_{p} / \partial u^{1}, \cdots, \partial \phi_{p} / \partial u^{d}\right)$, i.e., $P_{p}^{i}=\partial \phi_{p} / \partial u^{i}$. Then a normal vector at $u$ is $N_{p}=\left(P_{p}^{1}, \cdots, P_{p}^{d}, 0, \cdots, 0,1,0, \cdots, 0\right)$, where the 1 is the $(d+p)$ th coordinate of $N_{p}$. The vectors $N_{1}, \cdots, N_{\delta}$ are linearly independent. This makes it clear that if $P=\left(P_{1}, \cdots, P_{d}\right), J=(\partial P / \partial u)$, and $\rho(u)=$ rank $J(u)$, then the relative nullity $\nu(u)$ is $d-\rho(u)$.

Suppose, first, that $u_{0}=\psi\left(m_{0}\right)$ and that $\nu\left(u_{0}\right)=\nu^{*}\left(u_{0}\right)=\nu$. Suppose that (3.1) is defined on a $u$-domain $D$ containing $u_{0}$. Then, there is a $\nu$-plane section $\pi_{\nu}$ of $D_{\nu}$ through $u_{0}$ satisfying the conclusion of Lemma 2.1. Let $\pi_{\nu}$ be the intersection of $D_{\nu}$ and the $\nu$-plane (2.6) on which $v=\left(v^{1}, \cdots, v^{\rho}\right)$ is a constant, $\rho=d-\nu$.

In (3.1), consider the $C^{1}$ change of local coordinates $u \rightarrow v$ on $M$ given by (2.6). Then

$$
\partial x^{p} / \partial v^{k}=\left(\partial \phi_{p} / \partial u^{\alpha}\right)\left(a^{\alpha x}\right)+\partial \phi_{p} / \partial v^{k}
$$

Note that, by the proof of Lemma 2.1, $\partial \phi_{p} / \partial u^{\alpha}, \partial \phi_{p} / \partial v^{\alpha}$ are functions of $v^{1}, \cdots, v^{p}$. Hence the function $x^{p}$ is of the form

$$
x^{p}=a^{d+p, \kappa}\left(v^{1}, \cdots, v^{\rho}\right) v^{\alpha}+b^{d+p}\left(v^{1}, \cdots, v^{\rho}\right) \quad \text { for } p=1, \cdots, \delta,
$$

and so, (3.1) can be written as

$$
X=A_{\kappa}\left(v^{1}, \cdots, v^{\rho}\right) v^{\kappa}+B\left(v^{1}, \cdots, v^{\rho}\right),
$$

$X=\left(X^{1}, \cdots, X^{d+\delta}\right)$ and 


$$
\begin{aligned}
B & =\left(b^{1}, \cdots, b^{\rho}, 0, \cdots, 0, b^{d+1}, \cdots, b^{d+\delta}\right), \\
A_{\kappa} & =\left(a^{1 \kappa}, \cdots, a^{\rho \kappa}, 0, \cdots, 0,1,0, \cdots, 0, a^{d+1, \kappa}, \cdots, a^{d+\delta, \kappa}\right) .
\end{aligned}
$$

The " 1 " is the $x$ th component of $A_{\kappa}$. The vector functions $A_{\kappa}$ and $B$ are of class $C^{1}$.

The argument up to this point shows that (3.3) is valid in a neighborhood of the $\nu$-plane section $\pi_{v}$ of $D_{v}$. By the isometric property of the immersion $\psi$, the pre-image under $\psi$ of a line segment in the $X$-space of the form: $\left(v^{\alpha}=\right.$ const, $v^{\alpha}=$ linear function of $\left.t\right)$ is a geodesic. Hence a preimage of $\pi_{\nu}$ is a totally geodesic submanifold $M^{\nu}$ of $M(\nu)$.

If $\pi_{\nu}$ [and/or $M^{\nu}$ ] has a limit point $u_{1} \in D_{\nu}$ [and/or $\left.m_{1}=\psi^{-1}\left(u_{1}\right) \in M(\nu)\right]$, then the normal space at $u_{1}$ is the same as that at $u_{0}$. Hence $\psi$ is given locally in the form (3.1). Also $\nu\left(u_{1}\right)=\nu^{*}\left(u_{1}\right)=\nu$ by Lemma 2.1.

This shows that $M^{\nu}$ has a (maximal) extension so that its boundary points, if any, are not in $M(\nu)$. This implies the lemma for the case $\nu\left(m_{0}\right)$ $=\nu^{*}\left(m_{0}\right)=\nu$.

If $\nu^{*}\left(m_{0}\right)=\nu^{*}$ but $\nu\left(m_{0}\right)>\nu^{*}$, then there exist points $m_{1}, m_{2}, \cdots$ of $M$ such that $m_{n} \rightarrow m_{0}$ as $n \rightarrow \infty$ and $\nu\left(m_{n}\right)=\nu^{*}\left(m_{n}\right)=\nu^{*}$. After a selection of a subsequence, if necessary, it can be supposed that the $\nu^{*}$-plane section $\pi_{\nu^{*}}\left(m_{n}\right)$ of $S(\nu)=\psi(M(\nu))$ passing through $\psi\left(m_{n}\right)$ tends to a limiting position (in a suitable sense) and has the desired properties.

Remark. If $M=M^{d}$ and its immersion $\psi$ are of class $C^{t+1}, t \geqq 1$, then, in the local coordinates $v$, the immersion $\psi$ given by (3.3) is of class $C^{t}$.

4. Of particular interest is the question as to whether or not the planes $\pi(m)$ above are parallel in $E^{d+\delta}$. A sufficient condition is given in the next lemma for the case that $\nu^{*}(m)$ is constant (near $m_{0}$ ) and $M^{\nu^{*}(m)}$ is complete, so that $\psi\left(M^{*}(m)\right.$ is an entire $\nu^{*}(m)$-plane.

In this situation, the problem is reduced to the consideration of a $d$ dimensional surface $S: X=X(v)$ in an $E^{d+\delta}$ space of points

$$
X=\left(X^{1}, \cdots, X^{d+\delta}\right) \text {, }
$$

where $X(v)$ is of the form (3.3) for small $\left|v^{1}\right|, \cdots,\left|v^{\circ}\right|$ and for arbitrary $v^{\rho+1}, \cdots, v^{d}$. In (3.3), $B$ and $A_{\rho+1}, \cdots, A_{d}$ are $(d+\delta)$-dimensional vectors and $\partial X / \partial v^{1}, \ldots, \partial X / \partial v^{d}$ are linearly independent. The problem is to give sufficient conditions to assure that, after a suitable change of coordinates leaving the form of (3.3) unchanged, the vectors $A_{k}\left(v^{1}, \cdots, v^{\rho}\right)$ are constant.

Lemma 4.1. Let $0<\nu<d$ and $\nu+\rho=d$. Let $S$ be a d-dimensional surface in $E^{d+\delta}$ of class $C^{2}$ having a $C^{1}$ parametric representation

$$
S: X=A_{\kappa}\left(v^{1}, \cdots, v^{\rho}\right) v^{\kappa}+B\left(v^{1}, \cdots, v^{\rho}\right)
$$

for small $\left|v^{1}\right|, \cdots,\left|v^{\rho}\right|$ and arbitrary $v^{\kappa}, \quad \kappa=\rho+1, \cdots, d$, such that the relative nullity $\nu(v)$ of $S$ at $v$ is the constant $\nu(v)=d-\rho$ and that all vectors 
$y=\left(0, \ldots, 0, y^{\rho+1}, \ldots, y^{d}\right)$ are in trivial asymptotic directions at $\nu$ (so that the normal space of $S$ is independent of $v^{\rho+1}, \ldots, v^{d}$; cf. Lemma 3.1). In addition, suppose that all 2-dimensional sections of $S$ have non-negative curvatures. Then there exists a $C^{1}$ nonsingular linear change of the $v^{*}$ variables depending on $\left(v^{1}, \cdots, v^{\circ}\right)$,

$$
v^{\kappa}=a_{\lambda}^{\alpha}\left(v^{1}, \cdots, v^{\rho}\right) w^{\lambda}+c^{\kappa}\left(v^{1}, \cdots, v^{\rho}\right),
$$

such that (4.1) becomes

$$
S: X=C_{\kappa} w^{\kappa}+D\left(v^{1}, \cdots, v^{\rho}\right),
$$

where $C_{\rho+1}, \cdots, C_{d}$ are constant vectors.

Proof. Let $A_{\kappa}=\left(A_{\kappa}^{1}, \cdots, A_{\kappa}^{d+\delta}\right)$. Since $\partial X / \partial v^{k}=A_{k}$, the vectors $A_{\rho+1}$, $\cdots, A_{d}$ are linearly independent. After a rotation of the $X$-space, if necessary, it can be supposed that the $\nu$ vectors with $\nu$ components given by $\left(A_{k}^{\rho+1}, \cdots, A_{k}^{d}\right)$ are linearly independent at $v^{\alpha}=0$, hence for small $\left|v^{\alpha}\right|$. Choose the function $a_{\lambda}^{\alpha}\left(v^{1}, \cdots, v^{\rho}\right)$ of class $C^{1}$, so that

$$
A_{\kappa}^{\tau}\left(v^{1}, \cdots, v^{\rho}\right) a_{\lambda}^{\alpha}\left(v^{1}, \cdots, v^{\rho}\right) \equiv \delta_{\lambda}^{\tau} .
$$

Thus, after the change of variables $v^{\alpha}=a_{\lambda}^{\alpha} w^{\lambda}$ and the renaming of $w^{\lambda}$ back to $v^{\lambda},(4.1)$ has the same form, where

$$
A_{\kappa}=\left(A_{\kappa}^{1}, \cdots, A_{\kappa}^{\rho}, 0, \cdots, 0,1,0, \cdots, A_{\kappa}^{d+1}, \cdots, A_{\kappa}^{d+\delta}\right)
$$

and the " 1 " is the $\kappa$ th component of $A_{k}, \kappa=\rho+1, \ldots, d$. If, in (4.1), $v^{*}$ is replaced by $v^{k}+c^{k}\left(v^{1}, \cdots, v^{p}\right)$, then (4.1) takes the form $A_{k} v^{*}+\left(B-A_{k} c^{k}\right)$. Thus, in view of (4.4), the functions $c^{\star}\left(v^{1}, \cdots, v^{0}\right)$ can be chosen of class $C^{1}$, so that the $\kappa$ th coordinate of $B-A_{\kappa} c^{\kappa}$ is 0 for $\kappa=\rho+1, \cdots, d$. Thus, if $B-A_{x} c^{x}$ is called $B$ again, it can be supposed that

$$
B=\left(B^{1}, \cdots, B^{\rho}, 0, \cdots, 0, B^{d+1}, \cdots, B^{d+\delta}\right) .
$$

After this normalization, it will be shown that $A_{\kappa}$ is a constant vector by virtue of the fact that $v^{*}$ is arbitrary in (4.1).

Note that

$$
X_{\kappa}=A_{\kappa}, \quad X_{\alpha}=A_{\alpha \alpha} v^{\kappa}+B_{\alpha},
$$

where $A_{\kappa \alpha}=\partial A_{\kappa} / \partial v^{\alpha}, \quad \alpha=1, \cdots, \rho, \quad$ and $\quad \kappa=\rho+1, \cdots, d$. The vectors

$$
B_{1}, \cdots, B_{\rho} \text { and } A_{\rho+1}, \cdots, A_{d}
$$

are linearly independent. The vectors $A_{x \alpha}$ are in the span of (4.7) since

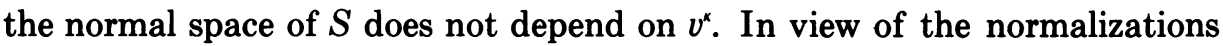
(4.4) and (4.5), it is clear that $A_{\kappa \alpha}$ is in the span of the set of vectors $B_{1}$, $\ldots, B_{\rho}$. Hence, the analogue of the Gauss equations give

$$
A_{\kappa \alpha}=\Gamma_{k \alpha}^{\beta} B_{\beta} \text {. }
$$

In view of the low differentiability of the parametrization of $S$ involved, 
it is best to consider the equations (4.8) as defining the continuous functions $\Gamma_{k \alpha}^{\beta}\left(v^{1}, \cdots, v^{\rho}\right)$, so that

$$
\Gamma_{\kappa \alpha}^{\beta}=g^{\beta \gamma} A_{\kappa \alpha} \cdot B_{\gamma},
$$

where $\left(g^{\beta \gamma}\right)=\left(g_{\beta \gamma}\right)^{-1}$ and $g_{\beta \gamma}=B_{\beta} \cdot B_{\gamma}$. It has to be verified that

$$
\Gamma_{\kappa \alpha}^{\beta}=0, \quad \text { i.e., } A_{\kappa} \text { is constant. }
$$

Since $S$ has local $C^{2}$ parametrizations, there exists a $C^{1}$ orthonormal basis $N^{1}\left(v^{1}, \cdots, v^{\rho}\right), \cdots, N^{\delta}\left(v^{1}, \cdots, v^{\rho}\right)$ for the normal space to $S$ at $X(v)$. The second fundamental matrix $\left(h_{i j}^{p}\right)$ corresponding to $N^{p}$ is given by $h_{i j}^{p}=-X_{i} \cdot N_{j}^{p}=-X_{j} \cdot N_{i}^{p}$, which is consistent with the tensor character of $\left(h_{i j}^{p}\right)$. The functions $h_{i j}^{p}$ will be considered as functions of $v^{\alpha}$ alone (with $v^{k}=0$ ).

Since all vectors $y=\left(0, \cdots, 0, y^{\rho+1}, \cdots, y^{d}\right)$ are in trivial asymptotic directions, so that $0=h_{i j}^{p} y^{j}=h_{i k}^{p} y^{k}$, it follows that $h_{i k}^{p}=0$ for $p=1, \cdots, \delta$, $i=1, \cdots, d$, and $\kappa=\rho+1, \cdots, d$.

It will be shown that if $\Gamma_{k}, H^{p}$ denote the matrices $\Gamma_{\kappa}=\left(\Gamma_{\kappa \alpha}^{\beta}\right), H^{p}=\left(h_{\alpha \beta}^{p}\right)$, where $\alpha, \beta=1, \cdots, \rho$, then

$$
\Gamma_{\kappa} H^{p}=\left(\Gamma_{\kappa} H^{p}\right)^{*}=H^{p} \Gamma_{k}^{*},
$$

if $\Gamma_{k}^{*}$ is the transpose of $\Gamma_{\kappa}$. If the parametrization (4.1) of $S$ is sufficiently smooth, (4.11) is a consequence of the Codazzi equations but can be deduced more simply and directly as follows: Since $N_{\alpha}^{p}=\partial N^{p} / \partial v^{\alpha}$ is a linear combination of the vectors $B_{1}, \cdots, B_{\rho}$ and $N^{1}, \cdots, N^{\delta}$, it is easy to see that the following analogue of the derivation formulae of Weingarten hold

$$
N_{\beta}^{p}=-g^{\alpha \gamma} h_{\alpha \beta}^{p} B_{\gamma}+d_{\beta q}^{p} N^{q},
$$

where $0=h_{\alpha \beta}^{p}=-N_{\beta}^{p} \cdot A_{\kappa}$ and these derivation formulae define $d_{\beta q}^{p}$. Multiplying these relations scalarly by $A_{\kappa \alpha}$ and using (4.9) gives

$$
A_{\kappa \alpha} \cdot N_{\beta}^{p}=-\Gamma_{\kappa \alpha}^{\gamma} h_{\gamma \beta}^{p} \text {. }
$$

The left side is symmetric in the indices $\alpha, \beta$; in fact, the relations $-h_{\alpha \beta}^{p}$ $=X_{\alpha} \cdot N_{\beta}^{p}=X_{\beta} \cdot N_{\alpha}^{p}$ give the identity

$$
A_{\alpha \alpha} \cdot N_{\beta}^{p} v^{\alpha}+B_{\alpha} \cdot N_{\beta}^{p} \equiv A_{\alpha \beta} \cdot N_{\alpha}^{p} v^{\kappa}+B_{\beta} \cdot N_{\alpha}^{p},
$$

so that $A_{\kappa \alpha} \cdot N_{\beta}^{p} \equiv A_{\alpha \beta} \cdot N_{\alpha \cdot}^{p}$. This is equivalent to (4.11).

The fact that the relative nullity of $S$ is identically $\nu$ implies that if $x=\left(x^{1}, \cdots, x^{\rho}\right)$ is a $\rho$-dimensional vector, then

$$
H^{p} x=0 \text { for } p=1, \cdots, \delta \text { implies that } x=0 .
$$

The condition on the curvatures of 2 -sections of $S$ is equivalent to

$$
\sum_{p=1}^{\delta}\left[\left(H^{p} x \cdot x\right)\left(H^{p} y \cdot y\right)-\left(H^{p} x \cdot y\right)\left(H^{p} y \cdot x\right)\right] \geqq 0
$$


for all real vectors $x=\left(x^{1}, \cdots, x^{\rho}\right), \quad y=\left(y^{1}, \cdots, y^{\rho}\right)$.

In order to prove (4.10), it will first be shown that (4.11)-(4.13) has the following implications for $\Gamma=\Gamma_{k}$, fixed $\kappa=\rho+1, \ldots, d$ :

$$
\begin{gathered}
\text { the eigenvalues of } \Gamma^{*} \text { are real; } \\
\text { if } c=0 \text { is an eigenvalue of } \Gamma^{*} \text {, then the } \\
\text { corresponding elementary divisors of } \Gamma^{*} \text { are simple. }
\end{gathered}
$$

On (4.14). Let $\Gamma^{*} x=c x$ for some $x \neq 0$. Then $H^{p} \Gamma^{*} x=c H^{p} x$, so that $H^{p} \Gamma^{*} x \cdot x=c H^{p} x \cdot x$. Since $H^{p} \Gamma^{*}$ and $H^{p}$ are symmetric matrices, it follows that $c$ is real if $H^{p} x \cdot x \neq 0$ for some $p$. Note that (4.13) is assumed for real vectors $x, y$ but is then valid for real vectors $y$ and complex vectors $x$. Thus if $H^{p} x \cdot x=0$ for $p=1, \cdots, \delta$, it follows from (4.13) that $H^{p} x \cdot y$ $=0$ for $p=1, \cdots, \delta$ and for all real $y$ (hence, for all complex $y$ ). Consequently, $H^{p} x=0$ for $p=1, \cdots, \delta$. By (4.12), this implies that $x=0$ and gives a contradiction. Thus $H^{p} x \cdot x \neq 0$ for some $p$ and, consequently, $c$ is real. This proves (4.14).

On (4.15). Let $c=0$ be an eigenvalue of $\Gamma^{*}$ and suppose that there is a corresponding multiple elementary divisor. Then there is a vector $x$ such that

$$
\Gamma^{*} x=z \neq 0, \quad \Gamma^{*} z=0 .
$$

Then $H^{p} \Gamma^{*} z=0$, so that $\Gamma H^{p} z=0$. Hence $H^{p} z \cdot \Gamma^{*} y=0$ for all $y$ and $p=1, \cdots, \delta$. Choosing $y=x$ gives $H^{p} z \cdot z=0$ for $p=1, \cdots, \delta$. As above, this implies that $H^{p} z=0$ for $p=1, \cdots, \delta$ and hence $z=0$. This contradiction proves (4.15).

On (4.10). Suppose that $\Gamma=\Gamma_{x}$ is not 0 for some $\alpha$ at some point $\left(v^{1}, \cdots, v^{\rho}\right)$. Then by (4.14)-(4.15), $\Gamma^{*}$ has a nonzero, real eigenvalue, say, $-1 / c \neq 0$, and an eigenvector $\left(c^{1}, \cdots, c^{p}\right) \neq 0$, i.e.,

$$
c^{\alpha}\left(c \Gamma_{\alpha \alpha}^{b}+\delta_{\alpha \beta}\right)=0 \text { for } \beta=1, \cdots, \rho .
$$

In the second part of (4.6), choose $v^{\lambda}=0$ if $\lambda \neq \kappa\left(\kappa\right.$ fixed) and $v^{\kappa}=c$, so that

$$
c^{\alpha} X_{\alpha}=c^{\alpha}\left(c A_{\alpha \alpha}+B_{\alpha}\right) \text {; }
$$

by (4.8),

$$
c^{\alpha} X_{\alpha}=c^{\alpha}\left(c \Gamma_{\kappa \alpha}^{\beta}+\delta_{\alpha \beta}\right) B_{\beta}=0 .
$$

This contradicts the linear independence of $X_{1}, \cdots, X_{\rho}$ and shows that $\Gamma_{k} \equiv 0$. This completes the proof of Lemma 4.1.

\section{APPENDIX}

5. In view of the uses of Lemma 2 in [4] and of its generalization Lemma 2.1 above, it seems of interest to generalize it further. This appendix 
deals with a generalization in which "gradient maps" are replaced by "involutory systems."

Let $x=\left(x^{1}, \ldots, x^{d}\right), w=\left(w^{1}, \ldots, w^{d}\right)$ denote $d$-tuples of real numbers. The Poisson bracket $(F, G)$ of two real-valued functions $F(x, w), G(x, w)$ of class $C^{1}$ is defined to be

$$
\begin{aligned}
(F, G) & =\sum_{k=1}^{d}\left[\left(\partial F / \partial x^{k}\right)\left(\partial G / \partial w^{k}\right)-\left(\partial F / \partial w^{k}\right)\left(\partial G / \partial x^{k}\right)\right] \\
& =\sum_{k=1}^{d} \partial(F, G) / \partial\left(x^{k}, w^{k}\right) .
\end{aligned}
$$

A set $X=\left(X^{1}(x, w), \cdots, X^{d}(x, w)\right)$ of $d$ real-valued functions of class $C^{1}$ will be said to be an involutory system if the following two conditions hold:

$$
\begin{gathered}
\left(X^{i}, X^{j}\right) \equiv 0 \quad \text { for } i, j=1, \cdots, d, \\
\operatorname{rank}\left(\partial X^{i} / \partial x^{j}, \partial X^{i} / \partial w^{k}\right)=d ;
\end{gathered}
$$

cf. [1, Chapter 6]. In (5.3), $\left(\partial X^{i} / \partial x^{j}, \partial X^{i} / \partial w^{k}\right)$ is a matrix with $d$ rows $(i=1, \cdots, d)$ and $2 d$ columns $(j=1, \cdots, d$ and $k=1, \ldots, d)$.

The result to follow concerns $\delta$ involutory systems

$$
X_{p}=\left(X_{p}^{1}\left(x, y_{p}\right), \cdots, X_{p}^{d}\left(x, y_{p}\right)\right),
$$

where $p=1, \cdots, \delta$. For a fixed $p, X_{p}^{i}\left(x, y_{p}\right)$ is a function of $2 d$ real variables $\left(x, y_{p}\right)=\left(x^{1}, \ldots, x^{d}, y_{p}^{1}, \ldots, y_{p}^{d}\right)$; but in dealing with different values of $p, y=\left(y_{1}, \cdots, y_{b}\right)=\left(y_{1}^{1}, \cdots, y_{1}^{d}, y_{2}^{1}, \cdots, y_{\delta}^{d}\right)$ is considered as a set of $d \delta$ variables. For example, Lemma 2.1 concerns the $\delta$ involutory systems $X_{p}$ $=\left(X_{p}^{1}, \cdots, X_{p}^{d}\right)$, where

$$
X_{p}^{i}\left(x, y_{p}\right)=P_{p}^{i}(x)-y_{p}^{i} \quad \text { for } i=1, \cdots, d \text { and } p=1, \cdots, \delta .
$$

Let $D$ be an open set in the $(d+d \delta)$-dimensional $(x, y)=\left(x^{1}, \ldots, x^{d}\right.$, $\left.y_{1}^{1}, \ldots, y_{\delta}^{d}\right)$-space; $X=\left(X_{1}, \cdots, X_{\delta}\right)=\left(X_{1}^{1}, \ldots, X_{\delta}^{d}\right)$ a set of $d \delta$ functions $X_{p}^{i}\left(x, y_{p}\right)$ of class $C^{1}$ such that each function depends only on $2 d$ variables $\left(x, y_{p}\right)$ and, for a fixed $p=1, \cdots, \delta$, the set $X_{p}=\left(X_{p}^{1}\left(x, y_{p}\right), \cdots, X_{p}^{d}\left(x, y_{p}\right)\right)$ is an involutory system.

Let $J_{p}\left(x, y_{p}\right)$ be the $d \times d$ Jacobian matrix $J_{p}=\left(\partial X_{p}^{i} / \partial x^{j}\right)$, where $i, j$ $=1, \cdots, d$, and $J(x, y)$ the Jacobian matrix $J=(\partial X / \partial x)$, where $X$ $=\left(X_{1}, \cdots, X_{\delta}\right)=\left(X_{1}^{1}, \cdots, X_{1}^{d}, X_{2}^{1}, \cdots, X_{\delta}^{d}\right)$,

$$
J=\left(\begin{array}{c}
J_{1} \\
\vdots \\
J_{\delta}
\end{array}\right),
$$

so that $J$ has $d$ columns and $d \delta$ rows. For $(x, y) \in D$, let

$$
\rho(x, y)=\operatorname{rank} J(x, y) \text { and } \rho^{*}\left(x_{0}, y_{0}\right)=\limsup \rho(x, y)
$$


as $(x, y) \rightarrow\left(x_{0}, y_{0}\right)$. For a given integer $k$, let $S_{k}$ be the open subset of $D$ defined by

$$
S_{k}=\left\{(x, y) \in D: \rho^{*}(x, y) \leqq k\right\}
$$

and $S_{k}(y)$ the open set in $x$-space given by

$$
S_{k}(y)=\left\{x:(x, y) \in S_{k}\right\} .
$$

LEMma 5.1. Let $X_{p}^{j}\left(x, y_{p}\right)$ be do real-valued functions of class $C^{1}$ on an $(x, y)$-domain $D$ such that $X_{p}^{j}\left(x, y_{p}\right)$ depends only on $2 d$ real variables and $X_{p}$ $=\left(X_{p}^{1}\left(x, y_{p}\right), \cdots, X_{p}^{d}\left(x, y_{p}\right)\right)$ is an involutory system for $p=1, \cdots, \delta$. Let $\left(x_{0}, y_{0}\right) \in D$ have the property that $\rho\left(x_{0}, y_{0}\right), \rho^{*}\left(x_{0}, y_{0}\right)$ have a common value $\rho=d-\nu$. Then

$$
X\left(x, y_{0}\right)=X\left(x_{0}, y_{0}\right)
$$

on a unique $\nu$-dimensional plane section $\pi_{\nu}\left(x_{0}\right)$ of $S_{\rho}\left(y_{0}\right)$ through $x_{0}$; for points $x$ near $x_{0}$, (5.5) holds if and only if $x \in \pi_{\nu}\left(x_{0}\right)$; finally, $\rho\left(x, y_{0}\right)=\rho^{*}\left(x, y_{0}\right)$ for all $x \in \pi_{\nu}\left(x_{0}\right)$.

A "local" analogue of this lemma is known for the case $\delta=1$ (under slightly stronger differentiability conditions); cf. [1, pp. 95-96].

Proof. Let $\rho=\rho\left(x_{0}, y_{0}\right)=\rho^{*}\left(x_{0}, y_{0}\right)$. Without loss of generality, it can be supposed that the first $\rho$ columns of $J$ are linearly independent (so that each of the remaining $d-\rho$ columns of $J$ are linear combinations of the first $\rho$ columns) for $(x, y)$ near $\left(x_{0}, y_{0}\right)$. It will be shown that

(†) there exists a $\nu$-plane

$$
\pi: x^{\alpha}=\sum_{\kappa=\rho+1}^{d} a^{\alpha \kappa} x^{\kappa}+b^{\alpha}, \quad \alpha=1, \cdots, \rho,
$$

where $a^{\alpha \kappa}, b^{\alpha}$ are constants, such that $\pi$ passes through $x_{0}$ and that (5.5) holds on the connected component of $\pi \cap S_{\rho}\left(y_{0}\right)$ containing $x_{0}$; furthermore, for $x$ near $x_{0}$, (5.5) holds if and only if $x \in \pi$.

The local part of $(\dagger)$ will be deduced from Lemma 2.1 for arbitrary $\delta \geqq 1$ and some (essentially) known results on involutory systems.

Consider first one involutory system $X^{1}(x, w), \cdots, X^{d}(x, w)$ of class $C^{1}$ in a vicinity of a point $(x, w)=\left(x_{0}, w_{0}\right)$. Put

$$
z^{\prime}=X(x, w) \quad \text { and } \quad z_{0}^{\prime}=X\left(x_{0}, w_{0}\right) .
$$

(a) Suppose that

$$
\operatorname{det}\left(\partial X^{i} / \partial w^{j}\right) \neq 0 \quad \text { at }\left(x_{0}, w_{0}\right)
$$

then (5.7) has a unique solution for $w$ of class $C^{1}$,

$$
w=W\left(x, z^{\prime}\right)=\left(W^{1}, \cdots, W^{d}\right),
$$


for $\left(x, z^{\prime}\right)$ near $\left(x_{0}, z_{0}^{\prime}\right)$ and there exists a function $f\left(x, z^{\prime}\right)$ of class $C^{1}$ such that

$$
W^{i}=\partial f / \partial x^{i} \quad \text { for } i=1, \cdots, d .
$$

(It follows that $\partial f / \partial x^{i}$ is of class $C^{1}$, but $\partial f / \partial z^{\prime i}$ may only be continuous.)

The proof of this is a considerably simplified version of the arguments of $[1$, pp. 90-91]. In order to prove (a), it is sufficient to verify that

$$
\partial W^{i} / \partial x^{j}=\partial W^{j} / \partial x^{i} \quad \text { for } i, j=1, \cdots, d .
$$

To this end, substitute (5.9) into (5.7) and differentiate $X^{i}$ with respect to $x^{j}$ to obtain

$$
0=\partial X^{i} / \partial x^{j}+\sum_{k=1}^{d}\left(\partial X^{i} / \partial w^{k}\right)\left(\partial W^{k} / \partial x^{j}\right) .
$$

Multiply this relation by $\partial X^{m} / \partial w^{j}$ and add for $j=1, \cdots, d$,

$$
\begin{aligned}
& \sum_{j=1}^{d} \sum_{k=1}^{d}\left(\partial X^{i} / \partial w^{k}\right)\left(\partial W^{k} / \partial x^{j}\right)\left(\partial X^{m} / \partial w^{j}\right) \\
& =-\sum_{j=1}^{d}\left(\partial X^{i} / \partial x^{j}\right)\left(\partial X^{m} / \partial w^{j}\right)=-\sum_{j=1}^{d}\left(\partial X^{i} / \partial w^{j}\right)\left(\partial X^{m} / \partial x^{j}\right),
\end{aligned}
$$

where the last equality is a consequence of the fact that $X^{1}, \ldots, X^{d}$ is an involutory system. Let $T, S$ denote the matrices

$$
T=\left(\partial X^{i} / \partial w^{j}\right), \quad S=\left(\partial W^{i} / \partial x^{j}\right) .
$$

Then the expression on the left is the $(i, m)$ th element of the matrix product $T S T^{*}$. It follows that $T S T^{*}$ is a symmetric matrix. Since $T$ is nonsingular, it is seen that $S$ is symmetric, i.e., that (5.11) holds. This proves (a).

(b) Let $X=\left(X^{1}(x, w), \cdots, X^{d}(x, w)\right)$ be an involutory system as in step (a) and let

$$
r=\operatorname{rank}\left(\partial X^{i} / \partial x^{j}\right) \quad \text { at }\left(x_{0}, w_{0}\right)
$$

and let the columns $\partial X / \partial x^{i}$ be linearly independent for $i=1, \cdots, r$. Then

$$
\operatorname{det}\left(\partial X / \partial x^{1}, \cdots, \partial X / \partial x^{r}, \partial X / \partial w^{r+1}, \cdots, \partial X / \partial w^{d}\right) \neq 0 \text { at }\left(x_{0}, w_{0}\right)
$$

and $\Xi(\xi, \eta)=X(x, w)$, where

$$
\begin{gathered}
\xi^{\alpha}=x^{\alpha}, \quad \xi^{\kappa}=-w^{\kappa} \quad \text { and } \quad \eta^{\alpha}=w^{\alpha}, \quad \eta^{\kappa}=x^{\kappa} \\
\quad \text { for } \alpha=1, \cdots, r, \kappa=r+1, \cdots, d
\end{gathered}
$$

is an involutory system, i.e.,

$$
\sum_{k=1}^{d} \partial\left(\Xi^{i}, \Xi^{m}\right) / \partial\left(\xi^{k}, \eta^{k}\right)=0 \quad \text { for } i, m=1, \cdots, d .
$$


This follows from considerations of $[1, \mathrm{pp} .85-87]$.

Combining (a) and (b) gives:

(c) Let $X(x, w), z^{\prime}, x_{0}, w_{0}, r$ be as in (a), (b). Then there exists a $C^{1}$ real-valued function $f\left(x^{r+1}, \cdots, x^{d}, w^{1}, \cdots, w^{r}, z^{\prime}\right)$ of $2 d$ real variables such that (5.7) has a unique solution of class $C^{1}$ given by

$$
\begin{array}{ll}
x^{\alpha}=g^{\alpha}\left(x^{r+1}, \cdots, x^{d}, w^{1}, \cdots, w^{r}, z^{\prime}\right) & \text { for } \alpha=1, \cdots, r, \\
w^{\kappa}=h^{\kappa}\left(x^{r+1}, \cdots, x^{d}, w^{1}, \cdots, w^{r}, z^{\prime}\right) & \text { for } \kappa=r+1, \cdots, d,
\end{array}
$$

and

$$
g^{\alpha}=\partial f / \partial w^{\alpha}, \quad h^{\kappa}=-\partial f / \partial x^{\kappa}
$$

Thus, if

$$
w^{\prime i}=\partial f\left(x^{\alpha}, w^{\alpha}, z^{\prime}\right) / \partial z^{\prime i}
$$

then

$$
\sum_{i=1}^{d} w^{i} d z^{i}+\sum_{\alpha=1}^{r} x^{\alpha} d w^{\alpha}-\sum_{\kappa} w^{\kappa} d x^{\kappa}=d f .
$$

REMARK. It can be mentioned that if $w^{\prime}$ is made a function of $(x, w)$ by inserting (5.7) into (5.14), say,

$$
w^{\prime}=\left(W^{1}, \cdots, W^{d}\right), \quad \text { where } W^{i}=\partial f / \partial{z^{\prime}}^{i} \text { at } z^{\prime}=X(x, w),
$$

then

$$
z^{\prime}=X(x, w), \quad w^{\prime}=W(x, w)
$$

is a canonical transformation in the sense that

$$
w^{\prime} \cdot d z^{\prime}-w \cdot d x \text { is closed }
$$

(i.e., is locally a total differential of a function of class $C^{1}$ ). Note, however, that $W(x, w)$ in (5.15) may only be continuous. (This is a variant of the standard deduction of a $C^{1}$ canonical transformation from an involutory system of class $C^{2}$.)

(d) Let $X, z^{\prime}, x_{0}, w_{0}, r$ and $f$ be as in (c) and put

$$
F(x, w)=\sum_{\beta=1}^{n} x^{\beta} w^{\beta}+\frac{1}{2} \sum_{\lambda=r+1}^{d}\left(w^{\lambda}\right)^{2}-f\left(x^{\kappa}, w^{\alpha}, z_{0}^{\prime}\right),
$$

then $F(x, w)$ is of class $C^{2}$ and the equation

$$
X\left(x, w_{0}\right)=z_{0}^{\prime} \quad\left[=X\left(x_{0}, w_{0}\right)\right]
$$

is equivalent to

$$
\nabla F(x, w)=\nabla F\left(x_{0}, w_{0}\right)
$$

where 


$$
\nabla F=\left(\partial F / \partial x^{1}, \cdots, \partial F / \partial x^{d}, \partial F / \partial w^{1}, \cdots, \partial F / \partial w^{d}\right) .
$$

Actually, $F(x, w)=F\left(x, w ; z_{0}^{\prime}\right)$ and $F$ is a function of class $C^{1}$ in $\left(x, w, z_{0}^{\prime}\right)$. Assertion (d) is clear from the fact that (5.7) and (5.12) are equivalent and that (5.13), (5.16) give

$$
\begin{aligned}
& \partial F / \partial x^{\beta}=w^{\beta}, \partial F / \partial w^{\beta}=x^{\beta}-g^{\beta}\left(x^{\alpha}, w^{\alpha}, z_{0}^{\prime}\right) \text { for } \beta=1, \cdots, r, \\
& \partial F / \partial x^{\lambda}=h^{\lambda}\left(x^{\alpha}, w^{\alpha}, z_{0}^{\prime}\right), \partial F / \partial w^{\lambda}=w^{\lambda} \quad \text { for } \lambda=r+1, \cdots, d .
\end{aligned}
$$

(e) Note that, at $\left(x_{0}, w_{0}\right), \operatorname{rank}(\partial(\nabla F) / \partial w)=d$ and that $\operatorname{rank}(\partial(\nabla F) / \partial x)$ $=r$. In fact the $2 d \times d$ Jacobian matrix $(\partial(\nabla F) / \partial x)$ consists of a $d \times d$ zero matrix and a $d \times d$ matrix obtained by multiplying $(\partial X / \partial x)$ by a nonsingular matrix.

(f) Proof of the local part of $(\dagger)$. Define $x^{\prime}=\left(x_{1}^{\prime}, \cdots, x_{\delta}^{\prime}\right)$ by $x_{p}^{\prime}=X\left(x, y_{p}\right)$. Then, by (d), there exists a function $F_{p}\left(x, y_{p}\right)$ of class $C^{2}$ such that for $\left(x, y_{p}\right)$ near $\left(x_{0}, y_{p 0}\right)$,

$$
X_{p}\left(x, y_{p}\right)=x_{p 0}^{\prime} \quad\left[=X_{p}\left(x_{0}, y_{p 0}\right)\right]
$$

is equivalent to

$$
\nabla F_{p}\left(x, y_{p}\right)=\nabla F_{p}\left(x_{0}, y_{p 0}\right) \text {. }
$$

Also, $F_{p}\left(x, y_{p}\right)=F_{p}\left(x, y_{p} ; x_{p 0}^{\prime}\right)$ is of class $C^{1}$.

Consider $F_{p}\left(x, y_{p}\right)=F_{p}(x, y)$ to be a function of $d+d \delta$ variables $(x, y)$. Let $K_{p}(x, y)$ be the $(d+d \delta) \times(d+d \delta)$ matrix which is the Jacobian matrix $\partial\left(\nabla F_{p}\right) / \partial(x, y)$, where $\nabla F_{p}$ is the gradient of $F_{p}(x, y)$. Then, if

$$
\rho_{0}(x, y)=\operatorname{rank} K(x, y), \text { where } K(x, y)=\left(\begin{array}{l}
K_{1}(x, y) \\
\vdots \\
K_{\delta}(x, y)
\end{array}\right)
$$

and $\rho_{0}^{*}\left(x_{0}, y_{0}\right)=\lim \sup _{0}(x, y)$ as $(x, y) \rightarrow\left(x_{0}, y_{0}\right)$, it follows that $\rho_{0}\left(x_{0}, y_{0}\right)$ $=\rho_{0}^{*}\left(x_{0}, y_{0}\right)=\rho+d \delta$. In fact, if the first column of $K(x, y)$ is obtained by differentiating $\left(\nabla F_{1}, \nabla F_{2}, \cdots, \nabla F_{p}\right)$ with respect to $x^{1}$, the second with $x^{2}, \ldots$ and the last with $y_{\delta}^{d}$, then the first $\rho$ and last $d \delta$ columns of $K(x, y)$ are linearly independent. For the construction of $F$ in (d) shows that any linear homogeneous relation between the first $d$ columns of $K(x, y)$ is a consequence of the same relation between the columns of $J(x, y)$.

Thus, Lemma 2.1 implies that there exist constants $a^{\alpha k}, b^{\alpha}$ such that (5.5) holds for $x$ near $x_{0}$ if and only if $x$ is on the $\nu$-plane $\pi$ in (5.6). Furthermore, $a^{\alpha \kappa}, b^{\alpha}$ are $C^{1}$ functions of $\left(y_{0}, x_{0}^{\prime}\right)$.

(g) Completion of proof. It remains to prove the "in the large" assertion of Lemma 5.1. To this end, note that the analogue of condition (5.3) implies that the set of $d \delta$ variables $y=\left(y_{1}^{1}, \cdots, y_{\delta}^{d}\right)$ can be divided into two sets $v=\left(v^{1}, \cdots, v^{d \delta-\rho}\right)$ and $u=\left(u^{1}, \cdots, u^{\dot{\rho}}\right)$ such that, at $(x, y)=\left(x_{0}, y_{0}\right)$, 


$$
\operatorname{det}\left(\partial X / \partial x^{1}, \cdots, \partial X / \partial x^{\rho}, \partial X / \partial v^{1}, \cdots, \partial X / \partial v^{d \delta-\rho}\right) \neq 0 .
$$

Hence, the equations $x^{\prime}=X(x, y)$ can be solved locally for $x^{1}, \cdots, x^{\rho}$ and $v$ in terms of $x^{\prime}, x^{\rho+1}, \cdots, x^{d}$, and $u$ :

$$
\begin{array}{ll}
x^{\alpha}=g^{\alpha}\left(x^{\rho+1}, \cdots, x^{d}, u, x^{\prime}\right) & \text { for } \alpha=1, \cdots, \rho, \\
v^{\sigma}=h^{\sigma}\left(x^{\rho+1}, \cdots, x^{d}, u, x^{\prime}\right) & \text { for } \sigma=1, \cdots, d \delta-\rho,
\end{array}
$$

where $g^{\alpha}, h^{\sigma}$ are of class $C^{1}$.

The fact that $\rho\left(x_{0}, y_{0}\right)=\rho^{*}\left(x_{0}, y_{0}\right)$ implies that $h^{\sigma}=h^{\sigma}\left(u, x^{\prime}\right)$ does not depend on $x^{\rho+1}, \cdots, x^{d}$. Also, the local part of $(\dagger)$ implies that $g^{\alpha}$ is linear in $x^{\rho+1}, \cdots, x^{d}$ :

$$
\begin{aligned}
& x^{\alpha}=\sum_{\kappa=\rho+1}^{d} a^{\alpha \kappa}\left(u, x^{\prime}\right) x^{\kappa}+b^{\alpha}\left(u, x^{\prime}\right) \quad \text { for } \alpha=1, \cdots, \rho, \\
& v^{\sigma}=h^{\sigma}\left(u, x^{\prime}\right) \quad \text { for } \sigma=1, \cdots, d \delta-\rho,
\end{aligned}
$$

where $a^{\alpha \kappa}, b^{\alpha}$ and $h^{\sigma}$ are of class $C^{1}$. Since (5.22) is the inverse of $x^{\prime}=X(x, y)$ for fixed $x^{\rho+1}, \cdots, x^{d}$ and $u$,

$$
1=\operatorname{det}\left(\partial X / \partial x^{\alpha}, \partial X / \partial v^{\sigma}\right) \cdot \operatorname{det}\left(\partial\left(x^{\alpha}, v^{\sigma}\right) / \partial x^{\prime}\right)
$$

in obvious notation. It is clear from (5.22) that the second factor is bounded if $y=y_{0}$ (hence $u$ ) and $x^{\prime}=x_{0}^{\prime}$ are fixed and $x$ is bounded. Consequently, the completion of the proof of Lemma 5.1 is similar to that of Lemma 2.1 (or of Lemma 2 in [4]).

\section{REFERENCES}

1. C. Carathéodory, Variationsrechnung und partielle Differentialgleichungen erster Ordnung, Teubner, Leipzig, 1935.

2. S. S. Chern and N. H. Kuiper, Some theorems on the isometric embeddings of compact Riemann manifolds in Euclidean space, Ann. of Math. (2) 56 (1952), 422-430.

3. S. S. Chern and R. K. Lashoff, The total curvature of immersed manifolds, Amer. J. Math. 79 (1957), 308-318.

4. P. Hartman and L. Nirenberg, On spherical image maps whose Jacobians do not change sign, Amer. J. Math. 81 (1959), 901-920.

5. B. O'Neill, Isometric immersion of flat Riemann manifolds in Euclidean space, Michigan Math. J. 9 (1962), 199-205.

6. R. Sacksteder, On hypersurfaces with no negative sectional curvatures, Amer. J. Math. 82 (1960), 609-630.

\section{JOHNS HOPKINS UNIVERSITY,}

BALTimore, MARYLAND 